\title{
Suicide following acute traumatic spinal cord injury
}

\author{
F K Judd MD BS DPM FRANZCP ${ }^{1}$ D J Brown MBBS FRACP FACRM \\ ${ }^{1}$ Department of Psychiatry, ${ }^{2}$ Spinal Injuries Unit, University of Melbourne, Austin \\ Hospital, Heidelberg, Victoria 3084 Australia.
}

\begin{abstract}
The rate of suicide following spinal cord injury has not been extensively studied but appears to be greater than in the general population. Six patients who died by suicide, from a total of 342 patients who were treated for acute spinal cord injury over a 5 year period are described. Clinical features shared by this group of patients included being male; having schizoid, depressive or narcissistic personality traits; alcohol or drug abuse; family or significant others favouring death as a preferred option; and the development of significant depression.
\end{abstract}

Key words: spinal cord injury; depression; suicide.

\section{Introduction}

The psychological consequences and means of measuring adaptation to the disabilities and handicap of acute traumatic spinal cord injury (SCI) are poorly understood. The development of depression, the wish to die, non compliance with treatment, poor motivation with rehabilitation and various forms of self destructive behaviour including suicide may all be regarded as signs of poor adjustment.

Studies have shown that $50 \%$ of SCI patients describe suicidal thoughts ${ }^{1}$ but the actual frequency of suicide is unknown. Only a few follow up studies examining the frequency of suicide in SCI patients have been described. In one, $34 \%$ of 50 deaths recorded in 423 consecutive patients with SCI were attributed to suicide or to self neglect. ${ }^{2}$ In a second study of US veterans with spinal injuries during the period $1946-1965,1.2 \%$ died by suicide. ${ }^{3}$ It is suggested that the suicide rate is increasing. ${ }^{4}$

Two major groups of aetiological factors in suicide have been studied-sociological and illness related. Durkheim (1897) related suicide risk to the degree to which an individual is integrated into society. ${ }^{5} \mathrm{He}$ divided suicide into 3 types-anomic, egoistic and altruistic. Social isolation is often regarded as the most important social aetiological factor. Those most at risk are men 45-64 years old, women over 65 years old and single, widowed and divorced individuals. ${ }^{6}$ Studies of those who commit suicide indicate that more than $90 \%$ suffer from a psychiatric illness, most commonly depression (70\%) and alcoholism (15\%). ${ }^{6}$ The suicide rate for the phsyically disabled is higher than the general population. ${ }^{7}$

\section{The Austin experience}

The Austin Hospital Spinal Injuries Unit (SIU) accepts and treats all patients with acute traumatic SCI in Victoria, Tasmania and Southern New South Wales (population 5 million). Patients remain in the unit through both the acute and rehabilitation phases of treatment. Patients are then followed up (at 1, 3, 6, 12 months post discharge) while being rehabilitated into the community, at the hospital outpatient clinic or by the visiting nurse, and then seen at least annually for review. By this process, physical, psychological and social morbidity and the mortality rate of patients are monitored.

Between January 1984 and January 1989 a total of 342 patients admitted to the Austin Hospital Spinal Injuries unit were treated during the acute and active rehabilitation phases. All were seen for psychiatric assessment shortly after admission. Those felt to be at risk for poor psychological outcome were seen by a member of the psychosocial team for ongoing management and re- 
viewed by the liaison psychiatrist as appropriate. ${ }^{8}$ Here we describe 6 patients from this group of 342 who to August 1990 are known to have died from suicide or suspected suicide.

\section{Case 1}

A 22 year old single man was admitted following a motor bike accident in which he sustained a burst fracture of $\mathrm{C} 5$ resulting in a C6 incomplete/C8 complete quadriplegia. He was first seen for psychiatric assessment when concern was expressed due to lack of communication with staff and other patients. He was found to be an isolated young man who prior to the accident had lived with his parents and younger brother. $\mathrm{He}$ described that he had had difficulty making friends, and to cope with his social isolation had drunk alcohol to excess. He described intermittent use of marijuana. He had been unemployed for most of the time since leaving school and described his main interests as drinking. He expressed little motivation with respect to life goals.

$\mathrm{He}$ was felt to have marked schizoid (indifference to social relationships, enjoyed solitary activities, restricted range of emotional expression) and depressive personality traits. It was thought that in the past he had 'treated his depression' with alcohol. Whilst an inpatient in hospital he became significantly depressed. He was treated with antidepressant medication but was largely non compliant with this. He talked at length of his frustration and anger regarding his injury and expressed a wish that he would rather be dead than continue life with his disability. His parents also expressed the opinion that he would be better off dead. During his time in the unit his depression failed to improve. He was non compliant with antidepressant medication and reluctant to accept any psychotherapeutic intervention. Following his rehabilitation he was eventually discharged home to live with his parents. Shortly after discharge home he died as a result of a suicide/homicide pact with a close friend.

\section{Case 2}

A 51 year old married self employed labourer was admitted to the spinal injuries unit following a fall from a roof in which he sustained fractured vertebrae and spinal cord injury resulting in a T4 paraplegia. Whilst in the acute treatment phase of his injury he at times appeared depressed but was not considered to be suffering from a depressive illness. Subsequently whilst in the rehabilitation phase of his treatment he was noted to be anxious and irritable. He described extreme distress at the lack of privacy and intrusions that various aspects of his treatment, eg bladder and bowel care, caused. No symptoms of a depressive illness were detected. Marked denial regarding his disability was a prominent feature.

Following discharge from hospital he was seen in the outpatient clinic and noted to be moderately severely depressed. $\mathrm{He}$ was treated with the antidepressant Nomifensine, and appeared to respond to the antidepressant medication. However, he continued to express marked anger regarding his injury, and difficulty accepting his disability. It was noted that his disability had been exacerbated by the development of PAO limiting the range of movement in his left hip, necessitating the use of a reclining wheelchair. He expressed a persistent feeling that if he had to be looked after in this way (particularly have his bladder and bowel care done for him) then he might as well be dead. Twelve months after his last outpatient visit he died from a self inflicted gun shot wound to the head.

\section{Case 3}

A 27 year old single man was admitted to the spinal injuries unit following a diving accident in which he sustained a crushed fracture of $\mathrm{C} 4$ resulting in a complete $\mathrm{C} 4$ quadriplegia. Four months after admission to hospital he was seen for psychiatric assessment because of a stated wish to die and refusal of treatment. His wish to die appeared to be due to lowered mood, his frustration regarding his current situation and treatment, and his fears about his long term situation, ie being ventilator dependent. Assessment revealed he was a man with considerable drive and great physical ability who had succeeded in almost any sport to which he turned his hand. He described that he had always in the past achieved what he 
set out to do by exercising his will and his ability, and he expressed a clear desire not to continue to survive in his present state.

He was thought to be suffering from a moderately severe depressive illness and was treated with antidepressant medication. Over the next several weeks his mood improved with the antidepresants but his wish to die did not change. He remained an inpatient in hospital for 12 months and was subsequently discharged home (ventilator dependent) with attendant care. Some months later he died from unknown causes. Shortly prior to his death he had called his family to see him and had made 'final arrangements' consistent with suicidal intent.

\section{Case 4}

An 18 year old single man was admitted to the unit following a motor bike accident in which he sustained T12 fracture resulting in a complete T11 paraplegia. Routine assessment revealed personality and interpersonal problems. His parents had separated when he was aged 13 and he left home when aged 15 years. Since that time he had had a series of jobs and several short term relationships. He described that he often drank alcohol to excess and smoked marijuana.

Five weeks after admission he was noted to be severely depressed and expressed suicidal ideation. He was treated with the antidepressant Nomifensine and subsequently with Mianserin. Over this time his mood appeared to improve. However during his rehabilitation he expressed considerable anger and a tendency to blame others for his problems. Continuing depression did not appear to be of significant intensity. He described considerable difficulty in accepting his injury but persistently refused to participate in any psychotherapeutic work in order to deal with some of his non acceptance and angry feelings. Six weeks after discharge from hospital he died in an explosion in his garage. It was subsequently revealed by other patients that he had told them of his intention to kill himself following discharge.

Case 5

A 40 year old single self employed man was admitted following a motor vehicle accident in which he sustained a T5-6 dislocation resulting in a T6 paraplegia. When assessed he presented as an extremely ambitious man who had always gained enormous feelings of satisfaction from his ability to achieve in business and various hobbies, eg sailing around the world, flying and exploring. He was felt to have significant narcissistic (grandiose sense of self importance, preoccupied with fantasies of success and power, hypersensitive to the opinion of others) personality traits.

Two months after admission he was felt to be severely depressed. He described the belief that his future was doomed and became markedly socially withdrawn. He was initially reluctant to take antidepressant medication but subsequently agreed to do so. One week after commencing antidepressant medication whilst on weekend leave he electrocuted himself and died.

\section{Case 6}

A 20 year old single man was admitted with a T4 paraplegia sustained as a result of a gun shot wound. Originally from South East Asia, he had overstayed his visa and at the time of admission was in illegal immigrant. When initially assessed he was noted to be frightened and suspicious but had no obvious psychiatric disorder. Whilst an inpatient he was always reluctant to discuss his problems with other members of the general staff or the psychiatrist. It was noted that he expressed considerable concern regarding the way in which he would be ostracized by his community as a result of the (illegal) behaviour which led to the gun shot wound and a concern that he would not therefore be able to return to that society. He had few friends, no financial support and was uncertain as to whether he was to be dealt with by the police and/or deported.

During his time as an inpatient, although he was socially withdrawn and uncommunicative, he did participate in his rehabilitation and behaved appropriately when mixing with other patients. He acknowledged feeling frustrated about being confined to a wheelchair but denied feeling depressed or experiencing any depressive symptoms. 
When directly questioned about suicidal ideation he denied any suicidal intent. Six weeks after discharge from hospital he died as the result of an overdose.

\section{Discussion}

The rate of $6 / 342$ deaths by suicide in this group is much greater than for the general population, estimated for a similar time period in Australia to be per annum 16 per 100,000 for males and 6 for females. This increased rate has been noted previously in follow up studies of patients with SCI. ${ }^{2,3}$ It should be noted that this may be an underestimate of the suicide rate in this group. It is generally agreed that suicide is under-reported; deaths reported as accidents, misadventure or open verdict may account for many more suicides. The method of ascertaining and recording suicide as well as religious and social attitudes may all contribute to under-reporting.' Of note, cases 3 and 4 are assumed by us to be death by suicide, but were not reported officially as such. Furthermore, our rate of $6 / 342$ includes only those who died by overt and deliberate acts. Patients who died as the result of medical complications due to self neglect, alcohol and drug abuse considered by some as 'passive suicide' are not included. Obtaining accurate statistics for this group is extremely difficult.

All 6 patients who died by suicide were male. Three (cases 1, 4 and 5) were noted to have personality features associated with poor coping in the face of a traumatic life event or major loss. Two (cases 1 and 4 ) had low self esteem, poor interpersonal skills, previous difficulty coping with loss and had tended to use alcohol or drugs to deal with adversity or low mood. The third (case 5) relied on appearance and achievement for his self esteem. He was devastated by the realisation of his own vulnerability and loss of omnipotence and became severely depressed.

Five of the 6 patients developed moderately severe depression during their inpatient treatment. This is much greater than the rate of $20 \%$ previously reported for patients in our unit ${ }^{10}$ and consistent with previous studies noting the strong association between suicide and depression. ${ }^{6}$ Three (cases $2,3,4)$ responded to antidepressant medication with improvement of depressed mood and its associated symptoms. It was not possible to determine whether the suicide of case 2 occurred in the setting of recurrent depression, but the other 2 patients (cases 3 and 4) were thought not to be significantly depressed at the time of their death.

Only one patient (case 6) was not considered to be significantly depressed during his inpatient stay. This young man, faced with considerable legal and social problems, felt that as the result of his behaviour he would be unacceptable to his family and community. In this context, his death by overdose could be regarded as anomic suicide. ${ }^{5}$ Some members of the family and friends of 2 patients (case 1 and 3 ) openly expressed the opinion that the patient would be better off dead. In one case (case 3 ) friends attempted to help the patient commit suicide while in hospital, while the other patient (case 1) died in a suicide/homicide with a close friend, shortly after his discharge home.

This series of case reports involves only a small group of patients, but highlights a number of important features. The rate of suicide in this patient population is high. Clinical features shared by this group of patients who died by suicide included being male, premorbid personality features including schizoid, depressive or narissistic traits, a previous history of difficulty coping with loss, or the use of alcohol or drugs to deal with adversity, family or significant others favouring death as a preferred option, and the development of significant depression. A belief that they would not be able to adjust to life as a paraplegic or quadriplegic person was expressed by all 6 patients. However all except case 2 died within 6 months of discharge from hospital. Early intervention is important for those patients at risk of suicide. In particular, emphasis should be placed on the recognition and treatment of depression, facilitating coping in those with a past history of difficulty dealing with stress, and assisting family members as well as the patient to face the losses resulting from their injury. 


\section{References}

1 Pinkerton AC, Griffin ML (1983) Rehabilitation in females with spinal cord injury: a followup study. Paraplegia 21: 166-175.

2 Wilcox N, Stauffer E (1972) Followup of 423 consecutive patients admitted to the spinal cord centre Rancho Los Amigos Hospital, 1 January to 31 December 1987. Paraplegia 10: 115-122.

3 Nyquist R, Bors E (1967) Mortality and survival in traumatic myelopathy during nineteen years from 1946 to 1965. Paraplegia 5: 22-48.

4 Geisler WO, Jousse AT, Wynne-Jones M, Breithaupt D (1983) Survival in traumatic spinal cord injury. Paraplegia 21: 364-373.

5 Durkheim E (1897) Le Suicide. Alcan, Paris.

6 Barraclough B, Bunch J, Nelson B, Sainsbury P (1974) A hundred cases of suicide: clinical aspects. $B r J$ Psychiat 125: 355-373.

7 Hopkins M (1971) Patterns of self destruction among the orthopedically disabled. Rehabil Res Pract Rev 3: $5-16$.

8 Judd FK, Brown DJ (1988) The psychosocial approach to rehabilitation of the spinal cord injured patient. Paraplegia 26: 419-424.

9 Goldney RD, Burvill PW (1980) Trends in suicidal behaviour and its management. Aust NZ J Psychiat 14: 1-15.

10 Judd FK, Stone J, Webber JE, Brown DJ, Burrows GD (1989) Depression following spinal cord injury. A prospective inpatient study. Br J Psychiat 154: 668-671. 